\title{
NUOVE TECNOLOGIE, SOCIETÀ DELLA CONOSCENZA E “MENTE ORIZZONTALE"
}

\author{
Roberto Finelli ${ }^{1}$
}

\begin{abstract}
Sintesi:
La tesi di fondo è che le nuove tecnologie rappresentano un enorme sviluppo dell'umanità sul piano della trasmissione e dell'elaborazione delle informazioni. Ma questa gigantesca innovazione, per la cornice dell'accumulazione capitalistica in cui viene svolta e sviluppata, dà luogo ad una antropologia e ad una formazione della soggettività umana in cui il "conoscere" prevale e domina sul "sentire". La natura astratta della ricchezza del capitale, e la sua accumulazione, richiedono un individuo parimenti astratto, in cui la conoscenza diventa sempre più informazione. Questa fallace identificazione della conoscenza con l'informazione è basata su una epocale distorsione del sentire, per la quale il senso del conoscere non proviene più dal corpo emozionale e interiore del soggetto umano ma dall'esterno, secondo programmi e definizioni di senso in qualche modo già predefiniti e precompilati. In questo ambito di problemi il saggio utilizza una distinzione profonda di significato tra il lemma "tecnica" e il lemma "tecnologia", a partire da sollecitazioni presenti nelle pagine del Marx maturo, per mettere in discussione una contestualizzazione "umana" e non "capitalistica" della tecnologia. Nello stesso tempo l'autore svolge una critica profonda della concezione heideggeriana e della tecnica e del modo in cui le filosofie della postmodernità, soprattutto quelle della cosiddetta French Theory, sotto la guida di Nietzsche e di Heidegger, hanno registrato solo la superficializzazione del mondo messa in atto dalla nuova tipologia "flessibile" dell'accumulazione capitalistica. La liquidazione di ogni forma di soggettività operata dai pensatori francesi, se ha avuto il merito di criticare giustamente forme troppo identitarie e dogmatiche di soggettività, ha nello stesso tempo del tutto liquidato l'ipotesi marxiana del capitale come vero soggetto della società moderna e contemporanea, con la sua necessità intrinseca di creare un mondo sempre più omogeneo alla sua espansione e universalizzazione.
\end{abstract}

Parole chiave: Tecnologia, Marx, Heidegger, Teoria Francese, Psicoanalisi.

\section{NEW TECHNOLOGIES, KNOWLEDGE SOCIETY AND "HORIZONTAL MIND"}

\begin{abstract}
:
This article claims that the new technologies represent a huge development of humanity on the level of the transmission and processing of information. However, since the capitalistic accumulation provides the context for this innovation, this latter gives rise to an anthropology and a formation of human subjectivity which bestows upon "knowing" a primacy over "feeling". Given this starting point, my essay will use the semantic distinction between "technique" and "technology" to explore some notes of the late Marx, in order to question a "human" contextualization of technology, instead of a "capitalistic" one. At the same time, I will undertake a critique of Heidegger's conception of technique and of postmodern French philosophy, which is responsible for getting rid of Marx's hypothesis of the capital as the real subject of modern and contemporary society, with its intrinsic need to make a world more and more homogeneous with its own universalization.
\end{abstract}

Key-words: Technology, Marx, Heidegger, French Theory, Psychoanalysis.

1 Roberto Finelli (Roma 1945) conduce ricerche da molti anni sull'opera di Marx, di Hegel e di Freud. Ha composto, tra i vari suoi scritti, una trilogia: 1) Un parricidio mancato. Hegel e il giovane Marx, Torino 2004, dedicato ad illustrare i deficit antropologici di Marx e del marxismo (ed. ing. A Failed Parricide, Leiden 2016); 2) Un parricidio compiuto. Il confronto finale di Marx con Hegel, Milano 2015, che valorizza il Capitale come scienza imprescindibile della modernità; 3) Per un nuovo materialismo, Torino 2018, in cui utilizza l'opera di Spinoza e Freud per una nuova proposta etico-politica. Ha pubblicato anche una monografia sulla filosofia di Hegel, Mythos und Kritik der Formen. Die Jugend Hegels (1770-1803), Frankfurt a. M. 2000 (ed. it., Mito e critica delle forme. La giovinezza di Hegel, Lecce 2009). E-mail: roberto.finelli@uniroma3.it. 
1. Informazione e conoscenza.

L'ideologia sempre più dominante nella società contemporanea, io credo, sia quella che scambia la conoscenza umana con la trasmissione e l'elaborazione di informazioni. Sia cioè quella che confonde la costruzione di conoscenza come attribuzione di significato ad una sfera dell'esperienza e del mondo con la comunicazione di informazioni attraverso procedure e sistemi di segni.

D'altra parte è indubbio che le grandi trasformazioni nella storia della civilizzazione umana si sono sempre accompagnate a profonde rivoluzioni sul piano della comunicazione. L'invenzione dell'alfabeto ha consentito di poter sintetizzare l'enorme campo della comunicazione, sia orale che scritta, in soli 25/30 segni, consentendo all'umanità di uscire da una comunicazione iconografica o pittografica, il cui uso rimaneva nelle mani di pochi. L'alfabeto cioè ha rappresentato il mezzo fondamentale di passaggio da una società e da una cultura aristocratico-sacerdotale a una società di cultura potenzialmente accessibile a masse numerose di popolazione. L'altro grande passaggio nell'ambito dei sistemi di comunicazione è stato rappresentato certamente dall'invenzione della stampa e dall'abbandono della tecnica amanuense, con la diffusione enorme del libro e del documento scritto che ne è conseguita. Oggi stiamo vivendo, senza dubbio, la terza grande rivoluzione con le macchine informatiche, capaci di trasmettere ed elaborare una enorme quantità di segni condensate in una piccola unità di silicio.

Ma appunto l'ideologia contemporanea consiste nel vedere il mondo come un massive information process, all'interno del quale la stessa intelligenza umana viene considerata come una macchina computazionale che processa informazioni e che, per tale struttura di base, può essere sostituita dall'intelligenza artificiale, come macchine che possono elaborare una enorme quantità di segni $^{2}$.

Invece io credo che sia necessario mantenere la profonda differenza tra segno e simbolo, con la distinzione tra sintassi e semantica che essa comporta. Un sistema di segni segue infatti delle regole formali di connessione/disgiunzione, che ne costituiscono la grammatica, ad es. quelle di un codice binario e del rispetto del principio di non contraddizione. Tali regole formali di movimento dei segni sono appunto formali, perché formano una sintassi che è indipendente dal significato. Ed infatti segni alfabetici e numeri non esistono in natura. Mentre il simbolo è un segno che rimanda a un significato, ad una semantica, il cui senso orienta letteralmente la nostra vita, separando l'azione

2 Per una interpretazione critica di questa prospettiva cfr. G. Longo, 2019, Information at the Threshold of Interpretation Science as Human Construction of Sense, in A Critical Reflection on Automated Science - Will Science Remain Human?, (Bertolaso, Sterpetti eds), Springer. Berlin 2019.

\begin{tabular}{|l|l|l|l|l|}
\hline Qenista Dialectus & Ano 9 & n. 18 & Outubro 2020 & p. 350-364 \\
\hline
\end{tabular}


da seguire da quella da non seguire, il bene dal male, e costruendo in tal modo le intenzioni e le prospettive del nostro agire nel mondo.

Il significato dei segni in quanto simboli è dunque ciò che costituisce il contenuto non riducibile a linguaggio del nostro vivere e che affonda le sue radici nel corpo della nostra memoria e dei nostri sentimenti. Perché su memoria e sentimenti si costruiscono a poco a poco le prospettive secondo le quali diamo organizzazione e forma al mondo in cui viviamo: esattamente attraverso una memoria emozionale che seleziona le invarianti importanti nella nostra esperienza e lascia cadere ciò che è fuori del nostro interesse vitale ${ }^{3}$.

Questo significa dire, in una prospettiva materialistica, che si dà costruzione di conoscenza nell'essere umano solo quando il conoscere è connesso profondamente con il sentire, quale complesso di sentimenti che danno senso e che dirigono il nostro scambio, la nostra agency, rispetto al nostro ambiente biologico e sociale. Significa dire che nell'essere umano c'è una indispensabilità del corpo, fisico ed emozionale, nel costruire una conoscenza piena di senso. Significa dire che il cervello umano, come il cervello animale, forma l'informazione nel senso di conoscenza, attraverso un modo e dei percorsi che sono completamente diversi dal modo in cui quella stessa informazione verrà poi elaborata e formalizzata nei linguaggi binari del digital computer ${ }^{4}$.

2. Per tutto ciò l'Etica di Spinoza giganteggia nella storia della filosofia moderna. Perché ha connesso profondamente percorsi del corpo e percorsi del pensiero, perché è giunta per prima nella storia del pensiero occidentale a proporre una concezione materialistico-corporea della distinzione tra bene e male. Secondo Spinoza infatti quella distinzione fondamentale non nasce dal conoscere ma dal sentire. Una etica intellettualistica presuppone che bene e male sia oggetti esterni, appartenenti a una tradizione o a una struttura oggettiva della realtà. Mentre l'Etica di Spinoza giudica bene ciò che aumenta la potenza di vita del corpo emozionale dell'essere umano e produce un sentimento che chiama in latino laetitia: e giudica male ciò che diminuisce e rattrista quella stessa potenza di vita, generando il sentimento della tristitia. Il passaggio da un'etica intellettualistica del conoscere a un'etica materialistica del sentire credo sia ancora oggi

3 Su questo tema rimando alla trilogia del neurobiologo A. Damasio, Descartes' Error: Emotion, Reason, and the Human Brain, Grosset/Putnam, New York, 1994 (L'errore di Cartesio, tr. it. a cura di F. Macaluso, Adelphi, Milano 1995); The Feeling of What Happens: Body and Emotion in the Making of Consciousness, Harcourt, Orlando 1999 (Emozioni e coscienza, tr. it. a cura di S. Frediani, Milano Looking for Spinoza. Joy, Sorrow, and the Feeling Brain, Harcourt, Orlando 2003 (Alla ricerca di Spinoza. Emozioni, sentimenti e cervello, tr. it. a cura di I. Blum, Adelphi, Milano 2003).

4 Cfr. L. Oddo, L'Inconscio fra reale e virtuale, Moretti \& Vitali, Bergamo 2018.

\begin{tabular}{|l|l|l|l|l|}
\hline Q Ronista Oialectus & Ano 9 & n. 18 & Outubro 2020 & p. 350-364 \\
\hline
\end{tabular}


fondamentale per comprendere la profondità della compenetrazione di mente e corpo nell'esperienza umana e per comprendere .....5

Ma oggi la potenza dell'ideologia del mondo come massive information process è l'espressione della trasformazione epocale di un capitalismo, basato sulle nuove tecnologie informatiche, e caratterizzato da quella che D. Harvey ha definito «accumulazione flessibile» ${ }^{6}$. Il superamento dell'accumulazione di tipo fordista e la diffusione di una tipologia di lavoro autonomo, non fisso, con un alto grado di precarietà sta producendo infatti un nuovo tipo di antropologia individuale e collettiva.

Farsi capitale di se stessi, improntare la propria vita ad una mentalità quantitativocalcolante che, prima che a passioni e desideri, guardi ai costi e ricavi del proprio agire, introiettare la forma-capitale come accumulazione di competenze e abilità motivate assai più dal valore di scambio che non dal valore d'uso: queste appaiono oggi essere sono le richieste fondamentali che la produzione capitalistica legata alle nuove tecnologie informatiche avanza nei confronti della nuova forza-lavoro.

Questa nuova tipologia dell'umano, che vede una mente calcolante prevalere e dominare su un pensiero incarnato in affetti ed emozioni, configura un'antropologia costruita solo su un asse orizzontale di identità e relazione a scapito dello sviluppo dell'asse verticale, quale relazione interiore tra pensiero ed emozione ${ }^{7}$.

Tale antropologia dell'orizzontale, a causa della rimozione della dimensione verticale, rende impossibile ogni distanza critica da cui i processi di vita individuali e collettivi possano essere valutati e diretti. Essa rappresenta infatti la diffusione sull'intero corpo sociale di un comportamento manageriale, quale modo di agire in un mondo messo in connessione, organizzato attraverso la rete, in un mondo cioè orizzontale-rizomatico, dove la competenza primaria consiste nella capacità di entrare in relazione e costruire legami.

E' la produzione di una mente orizzontale, quale tipologia di mente diffusa e di massa, che dunque oggi è all'ordine del giorno quale funzione antropologica fondamentale per la produzione e la valorizzazione del capitale. Produzione di una mente orizzontale che testimonia, secondo la lezione di Marx, la tendenza della valorizzazione capitalistica a totalizzarsi all'intera vita sociale, togliendo in questo caso ogni distinzione tra vita privata e vita pubblica e giungendo a

5 Cfr. E. Scribano, La conoscenza del Bene e del Male. Dal Breve trattato all'Etica, in «Consecutio rerum», 2012, n. 2 (www.consecutio.org). Ma più in generale su Spinoza cfr. A. M. Matheron, Individu et communauté chez Spinoza, Éditions de Minuit, Paris 1988.

6 D. Harvey, The condition of Postmodernity, Balckwell, Oxford 1989 (La crisi della modernità, tr.it. a cura di M. Viezzi, Il Saggiatore, Milano 2015).

7 Ferrari, Lombardi

\begin{tabular}{|l|l|l|l|l|}
\hline Q Rovista Dialectus & Ano 9 & n. 18 & Outubro 2020 & p. 350-364 \\
\hline
\end{tabular}


plasmare secondo l'astrazione propria della ricchezza del capitale la stessa interiorità dell'essere umano.

\section{Il mito della società della conoscenza.}

Il mito della società contemporanea di essere una società della conoscenza e della partecipazione creativa di tutti al mondo inteso come massive information process è centrale in questo processo.

L'ideologia fondata sullo scambio tra conoscenza e informazione mette in scena infatti ancora una volta l'apparenza e la superficie di un lavoro concreto che nasconde la realtà di un lavoro astratto.

Si dice infatti che le nuove tecnologie informatiche abbiano bisogno di prestazioni lavorative sempre più prossime all'agire comunicativo e dunque di un soggetto capace di interagire con il suo ambiente lavorativo attraverso tutte le sue doti di intelligenza e riflessività, di autonoma capacità di scelta. Secondo tale visione nell'economia flessibile del postfordismo i contesti di produzione e di mercato, per l'organizzazione a rete che li caratterizza, sono sempre più complessi e differenziati. Dunque tali che per essere coordinati rimandano alla complessità e all'elasticità proprie della mente umana. Per cui oggi ci sarebbe bisogno di prestazioni con forte partecipazione soggettiva e con un grado di individualizzazione che confuterebbe il discorso marxiano sul lavoro astratto, utilizzabile ormai solo al passato per il capitalismo ottocentesco e novecentesco. Con la società postfordista e postindustriale sarebbe aumentata dunque, secondo questa visione, e si sarebbe estesa ormai in larghissima misura l'efficacia del lavoro e del sapere concreto, la necessità dell'intervento personalizzato, rispetto a modelli standardizzati di comportamento.

Ma la verosimiglianza di questo discorso rifletta solo una realtà di superficie, che a ben vedere va rovesciata. A me sembra infatti che oggi sia il linguaggio alfa-numerico delle macchine informatiche che con i suoi codici binari, codici cioè semplicati ed altamente formalizzati, comanda l'intelligenza umana. $E$ ' il linguaggio informatico depositato nei programmi di elaborazioni dati che richiede un ambiente già semplificato e in grado di essere processato dal computer, che richiede cioè un ambiente a un grado assai basso di imprevedibilità. Né è un caso che i sistemi di intelligenza artificiale più efficienti sono quelli che operano all'interno di ambienti di lavoro assai semplificati ed omologati ai limiti delle loro procedure di calcolo ${ }^{8}$.

8 Cfr. L. Floridi, The Fourt Revolution. How the Infosphere is Reshaping Human Reality, Oxford University Press, Oxford 2014 (La quarta rivoluzione. Come l'infosfera sta trasformando il mondo, tr. it. a cura di M. Durante, Raffaello Cortina, Milano 2017).

\begin{tabular}{|l|l|l|l|l|}
\hline Qenista Dialoctus & Ano 9 & n. 18 & Outubro 2020 & p. 350-364 \\
\hline
\end{tabular}


Voglio dire insomma che il problema delle nuove tecnologie oggi non è tanto quello del fatto che esse, in un futuro anche prossimo, porrebbero fine al lavoro umano, con la minaccia di sostituire integralmente con l'automazione e l'Intelligenza Artificiale la mente umana. E' quello invece di una riduzione/conformazione del mondo-ambiente, lavoratori della mente compresi, secondo parametri prevalentemente di semplificazione e misurazione quantitativa, adatti a costituire i data base del massive information process. Il vero problema è cioè quello di una superficializzazione del mondo ridotto a campi di misurazione e valutazione solo quantitativa, che escludono criteri e parametri orientati al valore. Ovvero, per usare il linguaggio sociologico di Max Weber, criteri che sono validi per l'agire determinato allo scopo e non per l'agire orientato al valore, capace di discutere, confrontare e scegliere tra scopi.

E' un'intelligenza infatti quella richiesta dalle tecnologie informatiche che può certamente operare e scegliere tra più variabili ma utilizzando programmi che già in qualche modo predeterminano e obbligano il campo delle risposte possibili. Vale a dire che il campo di azione del lavoratore intellettuale può essere incomparabilmente più vario e polisemico di quello dell'antico lavoratore manuale, ma nello stesso tempo quel medesimo campo è strutturato secondo sintassi e schede di lavoro che, per quanto molteplici, rimandano a una semantica, a una scelta cioè di significati e scopi articolabili e innovabili all'interno di un orizzonte dato. Anche in questo senso di orizzonte storicamente dato e non superabile si può dire che il capitalismo flessibile e globale ha sempre più bisogno di una mente orizzontale.

Io credo dunque che si possa affermare che se nel fordismo il sistema MaschinereiArbeitskraft richiedeva l'uso di un corpo senza mente, oggi l'economia capitalistico postfordista, nei suoi luoghi di sviluppo più avanzati, richiede una mente senza corpo. Una mente che deve essere anaffettiva e decorporeizzata e nella quale si dà conoscere senza riconoscere: ossia acquisizione ed elaborazione di informazioni senza riconoscimento del significato e del valore emozionale (e dunque del valore etico-politico) dei loro contenuti.

\section{Tecnica e tecnologia.}

Ovviamente la produzione della mente orizzontale non ha luogo solo attraverso le tecnologie e le strutture produttive del capitalismo flessibile. La formazione della mente orizzontale richiede un’organizzazione finalizzata a tale scopo da tutti gli apparati ideologici della società contemporanea: dalla produzione di informazione da parte dei media giornalistici e televisivi all'intiera filiera dell'istruzione scolastica inclusa quella universitaria. Così In particolare nella

\begin{tabular}{|l|l|l|l|l|}
\hline Q Rovista Dialectus & Ano 9 & n. 18 & Outubro 2020 & p. 350-364 \\
\hline
\end{tabular}


formazione scolastica abbiamo assistito, almeno a partire dalla fine degli anni ' 70 del secolo scorso, ad una caduto radicale della Storicismo di contro all'affermazione generalizzata di una cultura del Pragmatismo. Ossia è venuta meno una cultura delle grandi scansioni storiche e dei passaggi epocali da una forma sociale all'altra - è venuta meno una cultura delle radici, del senso della memoria delle loro trasformazioni e rotture - per l'affermarsi di una educazione basata sull'efficienza pratica e sul problem solving, su come risolvere problemi di un immediato presente, il cui senso sta tutto nella sincronia della sua attualità che non nella diacronia delle sue cause profonde.

Tanto che la storia diviene, come ha ben illustrato Frederic Jameson in Postmodernism, solo una baracca di deposito per gli attrezzi di bricolage, dove ciascuno va per prendere quello che gli serve, per risolvere appunto il suo problema, senza considerazione alcuna della complessità simbolica e della totalità sociale in cui quegli attrezzi del passato erano collocati e da cui prendevano senso. La distruzione dell'Historismus è divenuta così il principio base su cui riorganizzare l'intero processo formativo delle nuove generazioni, le cui conoscenze vengono sempre più piegate nel verso delle competenze: ossia della capacità di fare ed agire, togliendo progressivamente ogni differenza significativa tra l'età dello studio e l'età del lavoro.

Di conseguenza non è certo un caso che la «tecnica» sia divenuta la categoria centrale e fondante del modo in cui l'essere umano si rapporta oggi alla realtà. Ossia che la tecnica, quale capacità dell'homo faber di elaborare e trasformare il mondo a suo vantaggio, sia divenuta la caratteristica dominante e più diffusa della Weltanschauung contemporanea. Visione del mondo come ideologia ovviamente, visto che la concezione dell'essere umano come homo technicus, come specie che compensa la sua fragilità rispetto alle altre specie viventi per mezzo della tecnica, percorre, secondo la modalità di un concetto generale e generico, tutta la storia della cultura occidentale, dal mito di Protagora narrato nell'omonimo dialogo di Platone all'antropologia filosofica di Helmut Plessner. L'essere umano non ha le caratteristiche e le abilità particolari e determinate proprie di ciascuna specie vivente, perché non possiede l'habitus istintuale e predeterminato delle altre specie, ma può integrare questa sua debolezza e incompiutezza ontologica attraverso il grande dispiegamento delle sue invenzioni tecniche che gli garantiscono un habitus sostanzialmente illimitabile e ad espansione infinita.

Secondo questa ideologia la tecnica è dunque una determinazione antropologica, assai prima che determinazione sociologica e sociale. Apparterrebbe all'essenza della specie umana, prima che alle diverse configurazioni del suo essere sociale: giacchè senza di essa in generale, quali

\begin{tabular}{|l|l|l|l|l|}
\hline QRovista Dialectus & Ano 9 & n. 18 & Outubro 2020 & p. 350-364 \\
\hline
\end{tabular}


possano essere poi le differenze che la connotano di sistema sociale in sistema sociale, la specie umana non si sarebbe riprodotta e non si riprodurrebbe.

Tanto che lo stesso discorso heideggeriano sulla tecnica, che è diventato egemone tra molti intellettuali negli ultimi quarant'anni, soppiantando il discorso marxiano sui processi di lavoro, è, a ben vedere, null'altro che una variante rovesciata di questo dispositivo ideologico e metastorico. Per il filosofo tedesco infatti la tecnica infatti trova la sua origine nell'oblio che l'essere umano compie della sua più vera natura. Essa nascerebbe dalla rimozione della differenza ontologica, cioè della distanza abissale che stringe insieme e separa radicalmente Sein e Dasein. Per cui l'essere umano, sottrattosi alla cura dell'Essere e della propria autenticità, si volgerebbe verso un mondo fatto solo di oggetti, riducibili solo ad enti della sua manipolazione e dunque della tecnica 9 .

Ma proprio nella questione della tecnica sta, per chi come noi appartiene a una tradizione di studi marxiani, una delle espressioni più rilevanti, per non dire la principale e la più insidiosa, del feticismo del capitale. Sta, potremmo dire, nella sovrapposizione di tecnica a tecnologia: se per tecnica intendiamo appunto la caratterizzazione metastorica e generica delle capacità dell'homo faber e per tecnologia l'applicazione della scienza ai processi di lavoro che caratterizza in modo peculiare la produzione nella storia del capitalismo.

Non è senza rilievo infatti, a proposito di questo problema, che Karl Marx abbia ben frequentato il significato economico e insieme politico di ciò che significava Technologie nella Germania del '700, avendo fatti estratti a Londra, com'è ben noto, dai testi di Joseph Beckmann.

La Technologie tedesca costituiva una delle materie di studio e di formazione del burocrate dell'era del cameralismo tedesco che doveva controllare e gestire i processi di produzione secondo una serie di procedure ordinate, prive di sprechi e di forte implicazione con lo studio scientifico della natura. La Technologie era cioè la disciplina economico-politica che, distinguendo tra competenza del burocrate cameralista e lavoratori impiegati e comandati come esecutori all'interno di un processo di lavoro scandito da necessità naturalistiche, maggiormente mediava scienze naturali e dominio burocratico-statuale ${ }^{10}$.

9 Per una critica della teoria heideggeriana della tecnica (limitatamente alla $\tau \dot{\varepsilon} \chi v \eta$ nel mondo greco antico) mi permetto di rinviare al mio saggio, La dottrina della verità senza la dottrina della città, in «Consecutio rerum», anno 3, n. 6, aprile 2019 (www.consecutio.org).

10 Su questo tema cfr. l'ampia produzione, di G. Frison, Linnaeus, Beckmann, Marx and the foundation of Technology. Between natural and social sciences: a hypothesis of an ideal type. First Part: Linnaeus and Beckmann, Cameralism, Oeconomia and Technologie, in History and Technology, 1993, vol. 10, pp. 139-160; Beckmann, Marx, Technology and Classical Economics, "History and Technology", 1993, vol. 10, pp. 161- 173. Ma si guardi dello stesso autore anche Technical and technological innovation in Marx, "History and Technology", 1988, vol. 6, pp. 299-324.

\begin{tabular}{|l|l|l|l|l|}
\hline Q Ronista Dialectus & Ano 9 & n. 18 & Outubro 2020 & p. 350-364 \\
\hline
\end{tabular}


Marx usa con una certa indistinzione negli scritti sul Capitale i termini di Technik e di Technologie, ma a mio avviso egli mantiene fortemente, soprattutto nel capitolo $13^{\circ}$ su "Macchine e Grande Industria" e nel Manoscritto 1871-73, il significato di derivazione cameralistica, per cui tecnologia stava a significare controllo e dominio sul processo di elaborazione dell'oggetto come, insieme, controllo e dominio dei fattori umani implicati nella produzione. Nello stesso tempo quel Marx abbandona invece, a mio avviso, un significato antropologico di tecnica e di prassi come proprietà intrinseche al genere umano che era stato molto operoso e presente sia nei Manoscritti economico-filosofici del '44 che nella teoria del materialismo storico dell'Ideologia tedesca.

La Technologie marxiana degli scritti sul Capitale con il passaggio dalla sussunzione formale alla sussunzione reale della forza-lavoro al capitale, concettualizza infatti un sistema Maschinerei-Arbeitskraft in cui la forza-lavoro è necessariamente subalterna alla processualità automatica delle macchine. Anche perché Marx abbandona qui una teoria antropocentrica della macchina di ispirazione smithiano-hegeliana, presente anch'essa nei suoi scritti precedenti, secondo la quale la macchina deriverebbe dalla divisione dei movimenti lavorativi dell'essere umano, che, attraverso una sempre maggiore parcellizzazione e semplificazione, alla fine potrebbero essere sostituiti dai movimenti della macchina. Per accedere invece ad una teoria del macchinario non più antropomorfa ma articolata secondo la tripartizione macchina motrice- macchina trasmettitricemacchina utensile in cui l'applicazione della scienza matematico-fisico della natura all'intero maccanismo sottrae alla prestazione umana ogni centralità ed ogni primato fondativo di senso ${ }^{11}$.

Ed è appunto tale realtà e concetto della "tecnologia", come produttrice di beni-merci e insieme di lavoro astratto e comunque subordinato al piano dell'impresa capitalistica, che vengono dissimulati e falsificati nella rappresentazione di superficie della tecnica come strumentazione macchinica, risorsa oggettiva e neutrale, a disposizione della specie umana. Quando cioè la tecnica viene intesa, in sostanza, come ciò che attiene alla relazione soggetto umano-oggetto naturale, senza far entrare in gioco le dinamiche delle relazioni soggetto-soggetto/i.

Tale intendimento feticistico e di superficie della tecnica è del resto alla base anche della narrazione heideggeriana della tecnica, in cui il dispositivo soggetto-oggetto viene mediato non da relazioni umane storiche e sociali ma dalla storia dell'Essere. Per cui il pastore dell'Essere ha finito col teorizzare che la dilatazione a dismisura della tecnica nel mondo contemporaneo non è dovuta alla storia di quella relazione sociale moderna che chiamiamo capitale quanto invece alla storia di un Essere che invia agli esseri umani, come loro destino, la dimenticanza di sé medesimo

11 Sulla questione di quanto nel Marx del materialismo storico, prima del Capitale, pesi fortemente una subordinazione della teoria delle macchine al modulo, ancora antropocentrico, della «divisione del lavoro», rimando alle pagine 163-209 del mio testo, Un parricidio compiuto. Il confronto finale di Marx con Hegel, Jaca Book, Milano 20015.

\begin{tabular}{|l|l|l|l|l|}
\hline Q Rovista Dialectus & Ano 9 & n. 18 & Outubro 2020 & p. 350-364 \\
\hline
\end{tabular}


quale vero fondamento dell'esistere, e di conseguenza la disposizione a trattare la realtà intera come fondo inesauribile e manipolabile di una soggettività umana, trapassata inautenticamente dalla triade Essere/Esserci/Ente alla diade Esserci/Ente.

Ora è indubbio che il feticismo come occultamento delle relazioni più profonde della realtà sociale a scene e a figure di superficie sia intrinseco al concetto marxiano di capitale. Ma io credo che il feticismo come è esposto nel famoso quarto paragrafo del primo capitolo sia ancora esso stesso troppo di superficie, ancora troppo legato alla sfera della circolazione e dello scambio delle merci. Invece, soprattutto in condizioni di sussunzione reale io credo che più che il feticismo della merce vada messo a tema un feticismo più profondo, che possiamo chiamare feticismo del capitale. Per il quale la ricchezza astratta del capitale, con la sua logica accumulativa, pervade e svuota di senso autonomo il mondo del concreto. Ma nello stesso tempo ne lascia sopravvivere solo una pellicola di superficie, che ha appunto la funzione di occultare e dissimulare la logica dell'astratto che invece profondamente lo muove dall'interno ${ }^{12}$.

\section{Postmodernità e mente orizzontale.}

Da questo gioco di dissimulazione del capitale sono stati giocati a mio avviso tutti i pensatori del postmoderno che hanno criticato ogni uso possibile di categorie come sistema e totalità, verità e realtà: denunciando che erano solo categorie ideologiche appartenenti alle grandi narrazioni autocelebrative della modernità. La maggior parte dei pensatori del postmoderno si sono riconosciuti in una ermeneutica del frammento, dell'evento, dell'episodico, hanno rinunciato, per principio, ad ogni interpretazione della realtà istituita sul nesso di apparenza ed essenza, esteriorità ed interiorità, significante e significato, e si sono trovati a frequentare di conseguenza solo la superficie dell'agire sociale. Senza rendersi conto che in questo modo essi finivano per incontrare inconsciamente proprio il processo capitalistico di superficializzazione della realtà, per il quale le strutture profonde e strutturale della realtà cedono la scienza alla presenza solo di homines aconomici calcolanti e scambianti, a cose-merci oggetti di un consumo generalizzato, a eventi giornalieri e mutevoli ${ }^{13}$.

12 Su tale processo di superficializzazione della realtà nella società contemporanea il riferimento obbligato è a F. Jameson, Postmodernism: Or, the Cultural Logic of Late Capitalism, Duke University Press, Durham 1991 (Postmodernismo ovvero La logica culturale del tardo capitalismo, tr. it. di M. Manganelli, Fazi, Roma 2007).

13 Su tale tema, della forte esposizione delle culture della postmodernità ad una prospettiva del "fuori”" cfr. M. Gatto, Marxismo culturale. Estetica e politica della letteratura nel tardo Occidente, Quodlibet, Macerata 2012; Id., Resistenze dialettiche. Saggi di teoria della critica e della cultura, manifestolibri, Roma 2018.

\begin{tabular}{|l|l|l|l|l|}
\hline QRovista Dialectus & Ano 9 & n. 18 & Outubro 2020 & p. 350-364 \\
\hline
\end{tabular}


Gli eroi eponimi della postmodernità, ossia gli eroi che danno il nome a un intero periodo filosofico, sono stati rispettivamente F. Nietzsche e M. Heidegger. Sono loro infatti che hanno concepito i princìpi fondamentali della decostruzione della modernità. Nietzsche ha negato l'idea di ogni struttura di permanenza e di ogni forma di identità, teorizzando una filosofia della vita come inesausto, sempre diverso e mai prevedibile, divenire. Critico di ogni luogo del consistere, di ogni istituzione, do ogni valore, di ogni soggettività, per Nietzsche la vita è solo un confronto costantemente diverso tra forze di vita e forze di non-vita. L'eterno ritorno è appunto il sempre nuovo disporsi di questo parallelogramma di forze. Il superuomo è colui che riesce ad accettare questo riconfigurarsi sempre nuovo della vita e riconoscerlo come proprio. Da questo rifiuto di ogni organizzazione e struttura permanente è nato l'anarchismo rizomatico di Deleuze e la microfisica del potere di Foucault, accomunati dalla celebrazione di Nietzsche di contro alla metafisica marxista della storia e alla negazione di ogni soggettività autocentrata.

Ma è soprattutto alla rivoluzione reazionaria di Heidegger che si deve la fondazione della postmodernità e il congedo da ogni forma di soggettività, con la reintroduzione autoritaria di una categoria filosofica così arcaica e consumata come quella di Essere. Attraverso l'Essere Heidegger ha introdotto infatti nell'essere umano una profondità abissale, un fondo sfondato, un fondo senza fondo. In tal modo con la differenza ontologica tra Essere ed Esserci Heidegger ha fatto dell'essere umano un'esistenza sfondata e dunque, per definizione, indefinibile e indeterminabile. Ossia, per meglio dire, il fondamento sfondato dell'Essere, la sua intrinseca Alterità rispetto all'Esserci, obbliga a dire che l'essenza dell'essere umano consiste nell'a/Alterazione, nel farsi a/Altro rispetto alla propria quotidianità e a un'esistenza di base materialistica, fatta di bisogni e scambio organico con la natura e gli altri esseri umani. Il risultato è, a ben vedere, il medesimo di quello di Nietzsche: la identificazione della soggettività umana con il disvalore per eccellenza a fronte di un «Altrove» (per Nietzsche la vita, per Heidegger l'Essere), che vieta ogni autonomo strutturarsi della società e della storia. Entrambi muovono da un «Fuori»: Nietzsche dall'interiorità di un corpo emozionale che viene dilatato ad unica e vera cornice dell'accadere, Heidegger dall'immanenza di un Essere che in effetti è assoluta lontananza tanto da manifestarsi solo velandosi e nascondendosi.

Ma è proprio qui, in questo sgomento ed orrore di fronte all'idea della soggettività, io credo si collochi la genesi, sul piano propriamente filosofico, della mente orizzontale. Perché in queste teorie di un soggetto senza identità, senza un fondamento specifico e proprio, senza una storia familiare, senza una radice storica e sociale, nasce a ben vedere un essere umano

\begin{tabular}{|l|l|l|l|l|}
\hline Q Povista Dialectus & Ano 9 & n. 18 & Outubro 2020 & p. 350-364 \\
\hline
\end{tabular}


indeterminato $^{14}$ : ossia un essere umano privo di caratteristiche e pronto nella sua indeterminatezza ad essere determinato secondo protocolli comunicativo-linguistici, secondo dimensioni sempre più intersoggettive e sempre meno infrasoggettive ${ }^{15}$.

Per altro non si può certo negare che la decostruzione della modernità messa in atto dalla French Theory, non abbia avuto profondi meriti nel criticare e decostruire costruzioni di una soggettività troppo facilmente presupposta: a partire, in campo marxista, da una collettività di classe troppo immediatamente presupposta come soggetto di una prassi rivoluzionaria. In tal senso i pensatori postmoderni hanno positivamente continuato la demistificazione della ragione occidentale già intensamente iniziata, dopo Nietzsche, dalla Dialettica dell'Illuminismo di Horkheimer e Adorno. Così come in particolare Derrida ha condotto una critica meritoria, da cui non si può più tornare indietro, di qualsiasi chiusura identitaria difensiva ed escludente, così come di comunitarismi e fondamentalismi privi di ogni sensibilità all'ideale unificazione kantiana del genere umano. Per cui non v'è dubbio che tutte le filosofie della decostruzione abbiano contribuito ad un progresso, prezioso quanto mai irrinunciabile, riguardo alla sfera dei diritti di riconoscimento di minoranze etniche, religiose, di genere, di accoglienza di profughi e migranti, di estensione dei più elementari diritti umani.

Ma oltre questo decentramento della ragione occidentale la critica postmetafisica della ragione e del soggetto non è riuscita ad andare, obbligata a un pensiero negativo-distruttivo a cui era fatto divieto, per fondazione originaria, il poter svolgersi in una ricostruzione positiva del tema della soggettività, individuale e collettiva. Foucault, Deleuze e Derrida, insieme con la nozione di un'essenza metafisica dell'uomo, hanno disgregato e impedito ogni possibile forma di identità soggettiva, delineando al massimo, nella loro estremizzazione dissolutoria, un'antropologia aperta e atta al globale ma priva di ogni determinazione locale: dove ogni «proprio» è solo un «improprio» ${ }^{16}$. E proprio in questa negazione radicale del "locale" i pensatori della decostruzione hanno, a mio avviso, spianato la strada alla soggettività priva di ogni costituzione e di ogni biografia personale e pronta perciò alla nuova orizzontalità capitalistica della mente.

\section{Riconoscimento dell'altro e riconoscimento del sé.}

14 Cfr. F. Fistetti, Le ambivalenze e i paradossi della rivoluzione dei diritti: una storia filosofica e politica (saggio di prossima pubblicazione: In «Consecutio rerum», anno 3, n. 7, www.consecutio.org).

15 Sul tema di una sopravalutazione dell'intersoggettività rispetto a quella dell'infrasoggettività nelle scienze umane contemporanee, sia sociologiche che psicologico-psicoanalitiche cfr. R. Lombardi, Formless Infinity. Clinical Explorations of Matte Blanco and Bion, Routledge, London 2015. Id, Metà Prigioniero, Metà Alato, Bollati Boringhieri, Torino 2016.

16 Cfr. F. Fistetti, Le ambivalenze e i paradossi della rivoluzione dei diritti: una storia filosofica e politica, op. cit..

\begin{tabular}{|l|l|l|l|l|}
\hline Q Ronita Dialectus & Ano 9 & n. 18 & Outubro 2020 & p. 350-364 \\
\hline
\end{tabular}


Stiamo vivendo, come dicevo all'inizio, una rivoluzione epocale nelle tecnologie dell'informazione e della Intelligenza Artificiale che hanno già cambiato e continueranno a cambiare profondamente la qualità delle nostre vite. Sottrarsi oggi al loro uso e alla loro diffusività a gestione capitalistica appare null'altro che una lontana e disarmante utopia.

Ma nello stesso tempo non bisogna dimenticare le indicazioni antropologiche ed eticopolitiche che nascono da un nuovo materialismo che rilegge il vecchio materialismo marxiano in un modo originale e legato alla nostra attuale problematica esistenziale e sociale. Questo nuovo materialismo che fa del corpo, della sua natura biologica e della dinamica pulsionale che ne consegue, il principio di senso dell'essere umano può infatti aiutare a ricostruire il quadro di una nuova configurazione e di una nuova valorizzazione della soggettività da far entrare in campo contro la tipologia egemone oggi e sempre più diffusa di soggettività orizzontale. E' un nuovo materialismo che, oltre al marxismo, si riferisce alla migliore tradizione psicoanalitica d'ispirazione freudiana, rifiuta il lacanismo, e guarda soprattutto alla scuola psicoanalitica inglese di prima e seconda generazione (Klein, Winnicott, Bion). Di contro ad una tipologia di soggettività informatica, nella quale v'è separazione e scissione tra informazione ed emozione, tra schede di lavoro su programmi alfa-numerici e percezioni sensoriali - insomma di contro ad una umanità in cui corpo e mente devono escludersi a vicenda - il nuovo materialismo rivendica il diritto alla superiorità dell'interpretazione sull'informazione e che interpretazione significhi, non elaborazione digitale di informazioni, ma costruzione di conoscenza, ossia una selezione di scelte e di modalità di azione nei confronti del mondo..

Questo a mio avviso significa che nell'ambito di una nuova stagione dei diritti ciò che va rivendicato è un nuovo diritto, di massa, alla pratica e alla necessità del riconoscersi, ossia alla possibilità per ognuno di entrare in contatto con il proprio mondo emozionale e dunque di stabilire una connessione tra somatico e psichico.

Si è molto parlato e discusso negli ultimi decenni, ed anche a ragione, dei diritti del riconoscimento dell'altro, del suo trapassare da una condizione di esclusione a quella di inclusione in una cittadinanza economica, civile e politica, sempre più estesa e partecipata. Ma anche il diritto al riconoscimento non può rimanere confinato su un piano solo orizzontale. Perché, accanto al riconoscimento dell'altro, ciò che va salvaguardato e sempre più curato ed educato, è il riconoscimento del sé, il diritto di ciascuno a riconoscere sé medesimo, nel vero che si diceva di una soggettività che matura un alto grado di gravitazione interna, quanto a capacità di sentire il proprio sentire e di dare così senso al proprio essere nel mondo.

\begin{tabular}{|l|l|l|l|l|}
\hline Govista Dialectus & Ano 9 & n. 18 & Outubro 2020 & p. 350-364 \\
\hline
\end{tabular}


La civiltà giuridica del liberalismo e del kantismo ha iscritto nella storia dell'umanità il valore primario dell'autonomia, come libertà di pensare e di volere nel proprio foro interiore, indipendentemente da qualsiasi autorità e costrizione esterna. La civiltà giuridica del socialismo e del comunismo ha inscritto in quella stessa storia il valore primario dell'eguaglianza, come accessibilità per tutti ai beni primari della materialità dell'esistenza, e dunque come libertà dai bisogni elementari. Ma la nuova era delle tecnologie digitali e della mente orizzontale che l'accompagna introduce un nuovo imprescindibile valore: quello dell'individuazione. Ovvero quello della libertà, la più ampia possibile, per ciascuno di poter accedere, lungo l'asse interno della verticalità corpo-mente, alla propria irripetibile emotività e sensibilità: con il grado minimo di autorepressione e censura ${ }^{17}$.

Quali percorsi e quali istituzioni mettere in campo per fare della società del conoscere anche una società del riconoscere sarà, a mio avviso, il compito, assai difficile ma affascinante, delle generazioni future.

Bibliografia:

Damasio, A. Descartes' Error: Emotion, Reason, and the Human Brain, Grosset/Putnam, New York, 1994 (L'errore di Cartesio, tr. it. a cura di F. Macaluso, Adelphi, Milano 1995).

Damasio, A. The Feeling of What Happens: Body and Emotion in the Making of Consciousness, Harcourt, Orlando 1999 (Emozioni e coscienza, tr. it. a cura di S. Frediani, Milano, 1999).

Damasio, A. Looking for Spinoza. Joy, Sorrow, and the Feeling Brain, Harcourt, Orlando 2003 (Alla ricerca di Spinoza. Emozioni, sentimenti e cervello, tr. it. a cura di I. Blum, Adelphi, Milano 2003).

Harvey, D. The Condition of Postmodernity, Blackwell, Oxford 1989 (La crisi della modernità, tr.it. a cura di M. Viezzi, Il Saggiatore, Milano 2015).

Finelli, R. Un parricidio compiuto. Il confronto finale di Marx con Hegel, Jaca Book, Milano 2015.

Finelli, R. Per un nuovo materialismo. Presupposti antropologici ed etico-politici, Rosenberg \& Sellier, Torino 2018.

17 Anche qui, su questo tema e sula tragica assenza di una teoria dell'individuazione nel pensiero di Marx e nella tradizione marxista, mi permetto di rinviare al mio ultimo testo, Per un nuovo materialismo. Presupposti antropologici ed etico-politici, Rosenberg \& Sellier,Torino 2018, particolarmente pp. 213-227.

\begin{tabular}{|l|l|l|l|l|}
\hline Q Ronista Dialectus & Ano 9 & n. 18 & Outubro 2020 & p. 350-364 \\
\hline
\end{tabular}


Finelli, R. La dottrina della verità senza la dottrina della città, in «Consecutio rerum», anno 3, n. 6, aprile 2019 (www.consecutio.org).

Fistetti, F. Le ambivalenze e i paradossi della rivoluzione dei diritti: una storia filosofica e politica, In «Consecutio rerum», anno 3, n. 7, 2019 (www.consecutio.org).

Floridi, L. The Fourt Revolution. How the Infosphere is Reshaping Human Reality, Oxford University Press, Oxford 2014 (La quarta rivoluzione. Come l'infosfera sta trasformando il mondo, tr. it. a cura di M. Durante, Raffaello Cortina, Milano 2017).

Gatto, M. Marxismo culturale. Estetica e politica della letteratura nel tardo Occidente, Quodlibet, Macerata 2012.

Gatto, M. Resistenze dialettiche. Saggi di teoria della critica e della cultura, manifestolibri, Roma 2018.

Jameson, F. Postmodernism: Or, the Cultural Logic of Late Capitalism, Duke University Press, Durham 1991 (Postmodernismo ovvero La logica culturale del tardo capitalismo, tr. it. di M. Manganelli, Fazi, Roma 2007).

Lombardi, R. Formless Infinity. Clinical Explorations of Matte Blanco and Bion, Routledge, London 2015.

Lombardi, R. Metà Prigioniero, Metà Alato, Bollati Boringhieri, Torino 2016.

Longo, G. 2019, Information at the Threshold of Interpretation Science as Human

Construction of Sense, in: A Critical Reflection on Automated Science - Will Science Remain Human?, (Bertolaso, Sterpetti eds), Springer, Berlin 2019.

Matheron, A.M. Individu et communauté chez Spinoza, Éditions de Minuit, Paris 1988.

Oddo, L. L’Inconscio fra reale e virtuale, Moretti \& Vitali, Bergamo 2018.

Scribano, E. La conoscenza del Bene e del Male. Dal Breve trattato all'Etica, in «Consecutio rerum», n. 2, 2012, (www.consecutio.org).

\begin{tabular}{|l|l|l|l|l|}
\hline Q Povista Dialectus & Ano 9 & n. 18 & Outubro 2020 & p. 350-364 \\
\hline
\end{tabular}

